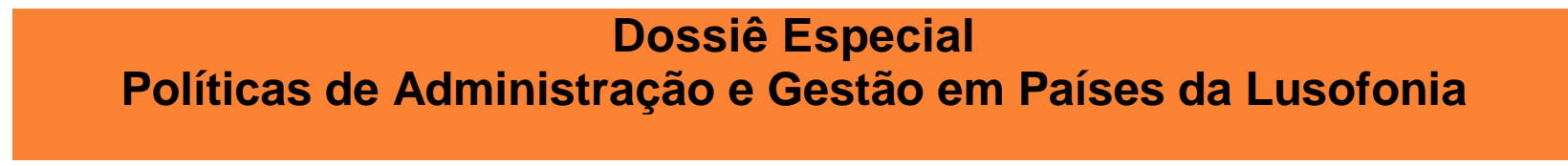

arquivos analíticos de políticas educativas

Revista acadêmica, avaliada por pares, independente, de acesso aberto, e multilíngüe

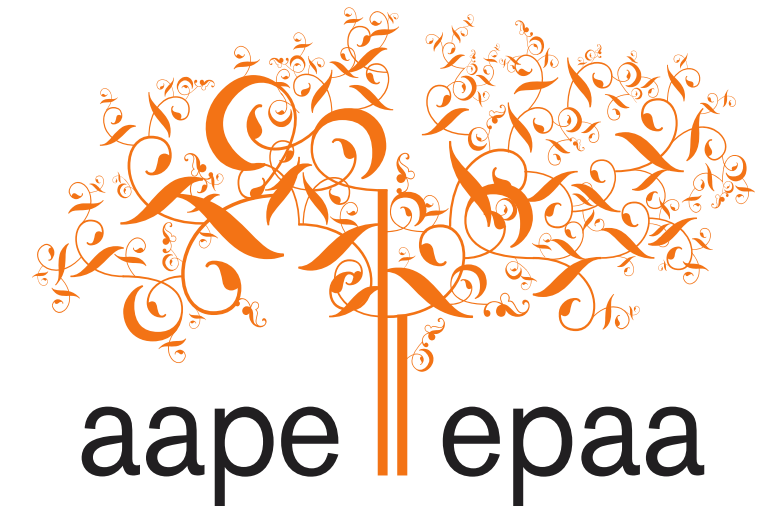

Arizona State University

\title{
Políticas de Administração e Gestão em Países da Lusofonia: Perspectivas Críticas sobre a Nova Gestão Pública e a Pós- burocracia em Educação
}

\author{
Almerindo Janela Afonso \\ Universidade do Minho \\ Portugal \\ ¿q \\ Geovana Mendonça Lunardi Mendes \\ Universidade do Estado de Santa Catarina \\ Brasil
}

Citação: Afonso, A. J., \& Mendes, G. M. L.. (2018). Políticas de administração e gestão em países da Lusofonia: Perspectivas críticas sobre a Nova Gestão Pública e a pós-burocracia em educação. Arquivos Analíticos de Políticas Educativas, 26(127). http://dx.doi.org/10.14507/epaa.26.4254 Este artigo faz parte do dossiê especial, Políticas de administração e gestão em países da Lusofonia: Perspectivas críticas sobre a Nova Gestão Pública e a pós-burocracia em educação, editada por Almerindo Janela Afonso e Geovana Mendonça Lunardi Mendes.

Resumo: Nas últimas décadas, ganhou centralidade a chamada Nova Gestão Pública/New Public Management que se tem traduzido, entre outras formas, pela incorporação e implementação de princípios de racionalidade empresarial e de gestão privada nas instituições e 
organizações públicas. Muitas reformas setoriais seguiram esses princípios, alguns dos quais foram concretizados na redefinição do papel do Estado e em alterações nos procedimentos da administração pública, tendo também induzido outras mudanças nos modos de regulação social em contexto de internacionalização crescente do capitalismo. $\mathrm{Na}$ sequência destas mudanças, a obsessão (frequentemente mais retórica do que real) com a eficácia e eficiência do Estado passou também a ser entendida no quadro de uma racionalidade pós-burocrática. Esta nova racionalidade foi, no entanto, pouco accionada nos textos que se seguem, tal como aconteceu com a da hiperburocracia-ao contrário do que era desejo dos editores deste dossiê. Esta constatação não deixa, de algum modo, de revelar que a ideologia da Nova Gestão Pública é ainda o referente hegemónico no campo da pesquisa e reflexão das ciências sociais e das ciências da educação. Ainda assim, do nosso ponto de vista, o que é mais notório nas contribuições que se seguem é que muitas das promessas das diferentes versões da ideologia da Nova Gestão Pública, ou não foram cumpridas, ou foram-no apenas parcialmente, sendo uma das críticas mais contundentes a esta ideologia aquela que se pode traduzir na ideia de que administrar é muito diferente de governar. Em síntese, o Dossiê aqui apresentado, objetiva, entre outros aspectos e em diferentes contextos nacionais da lusofonia, analisar reformas e políticas públicas de educação (com dominância em dimensões da administração e gestão, avaliação e accountability, privatização e mercadorização) que, implícita ou explicitamente, assumem, em graus muitos diversos de aprofundamento crítico e/ou de sustentação teórico-conceptual e empírica, alguns dos pressupostos atrás referenciados, os quais os/as autores/as entenderam priorizar.

Palavras-chave: Nova Gestão Pública; Administração e Gestão; Política Educacional

Management and management policies in Lusophone countries: Critical perspectives on the New Public Management and post-bureaucracy in education

Abstract: In recent decades, the New Public Management has achieved a central role. This centrality, among other things, is translated by the incorporation and implementation of the business rationale and private management in public institutions and organizations. Many sectorial reforms have followed these principles, some of which have been implemented in the redefinition of the role of the State and in changes in the procedures of public administration. Also, it induced other changes in the modes of social regulation in the context of the increasing internationalization of capitalism. Following these changes, the obsession (often more rhetorical than real) with the effectiveness and efficiency of the State is now understood in the framework of a post-bureaucratic rationality. However, the new rationality was little mobilized in the following texts, as happened with hyper bureaucracy - contrary to the wish this dossier's editors. This observation does not fail to reveal that the ideology of the New Public Management is still the hegemonic reference in the field of research and reflection in the field of social and education sciences. Nevertheless, from our point of view what comes as most striking in the contributions that follow is that many of the promises of the different versions of the New Public Management ideology have either not been fulfilled or were only partially achieved, representing the compelling criticism to the ideology summarized in the idea that managing is very different than governing. In conclusion, this dossier aims, among other aspects and in different Lusophony national contexts, to analyze reforms and public education policies (with a predominance of administration and management, evaluation and accountability, privatization and marketization), that implicit or explicitly, assume, to varying degrees of critic al deepening and/ or theoretical-conceptual and empirical support, some of the assumptions mentioned above, which the authors chose to prioritize.

Keywords: New Public Management; Administration and Management; Educational Policy 
Políticas de administración y gestión en países de la Lusofonía: Perspectivas críticas sobre la Nueva Gestión Pública y la post-burocracia en educación

Resumen: En las últimas décadas, ha ganado centralidad la llamada Nueva Gestión Pública/New Public Management que se ha traducido, entre otras formas, por la incorporación e implementación de principios de racionalidad empresarial y de gestión privada en las instituciones y organizaciones públicas. Muchas reformas sectoriales siguieron estos principios, algunos de los cuales se concretaron en la redefinición del papel del Estado y en cambios en los procedimientos de la administración pública, habiendo también inducido otros cambios en los modos de regulación social en contexto de internacionalización creciente del capitalismo. A raíz de estos cambios, la obsesión (a menudo más retórica que real) con la eficacia y eficiencia del Estado también se entiende en el marco de una racionalidad post burocrática. Esta nueva racionalidad fue, sin embargo, poco accionada en los textos siguientes, tal como sucedió con la de la hiperburocracia - al contrario de lo que era deseo de los editores de este dossier. Esta constatación no deja, de algún modo, de revelar que la ideología de la Nueva Gestión Pública es aún el referente hegemónico en el campo de la investigación y reflexión de las ciencias sociales y de las ciencias de la educación. Sin embargo, desde nuestro punto de vista, lo que es más notorio en las contribuciones que siguen es que muchas de las promesas de las diferentes versiones de la ideología de la Nueva Gestión Pública, o no se cumplieron, o lo fueron sólo parcialmente, siendo una de las críticas más contundentes a esta ideología aquella que se puede traducir en la idea de que administrar es muy diferente de gobernar. En síntesis, el Dossier aquí presentado, objetiva, entre otros aspectos y en diferentes contextos nacionales lusófonos, analizar reformas y políticas públicas de educación (con dominación en dimensiones de la administración y gestión, evaluación y accountability, privatización y mercantilización) que, implícita o implícita, en grados diversos de profundización crítica y/o de sustentación teórico-conceptual y empírica, asumen algunos de los presupuestos atrás referenciados, los cuales los/as autores/as entendieron priorizar.

Palabras-clave: Nueva Gestión Pública; Administración y Gestión; Política Educativa

\section{Introduzindo as Políticas de Administração e Gestão em Países da Lusofonia}

Nas últimas décadas, ganhou centralidade a chamada Nova Gestão Pública/New Public Management que se tem traduzido, entre outras formas, pela incorporação e implementação de princípios de racionalidade empresarial e de gestão privada nas instituições e organizações públicas. A Nova Gestão Pública pode mesmo ser considerada como um dos pilares estruturantes da agenda global para a educação, ainda que venha sendo materializada com especificidades próprias de cada contexto nacional (e.g. Verger \& Curran, 2014). Neste sentido, muitas reformas setoriais seguiram os seus princípios, alguns dos quais foram concretizados na redefinição do papel do Estado e em alterações nos procedimentos da administração pública, tendo também induzido outras mudanças nos modos de regulação social em contexto de internacionalização crescente do capitalismo. Uma das consequências imediatas, mais visíveis em certos países do que em outros, foi a retração das políticas públicas referenciáveis ao Estado Social (Welfare State). $\mathrm{Na}$ sequência da expansão daquela ideologia gestionária, e no que diz respeito mais especificamente à educação, a obsessão pelos resultados/produtos dos sistemas de ensino e a consequente secundarização ou invisibilização dos processos pedagógicos passaram a ser vistas como indutoras de eficácia e eficiência da ação do Estado e da administração pública, e esses resultados (coletados, nomeadamente, por formas de avaliação estandardizadas e analisados a posterior) começaram a ser entendidos no âmbito de uma 
nova forma de regulação pelo conhecimento ou regulação pós-burocrática (e.g. Barroso \& Carvalho). Poderse-á dizer, portanto, que este é um dos pontos-chave onde a nova gestão pública e a regulação pósburocrática convergem. No entanto, as promessas das diferentes versões da ideologia da nova gestão pública, ou não foram cumpridas, ou foram-no apenas parcialmente. Por um lado, existe evidência bastante que mostra que algumas formas e práticas que podem ser referenciadas à pós-burocracia não só substituíram, em certos casos, as formas e práticas características da burocracia, enquanto modelo tradicional de organização racional-legal, como coexistem com elas. Por outro lado, os desenvolvimentos da pesquisa empírica e teórico-conceptual, na esteira de leituras ou releituras mais cautelosas e sistemáticas de Max Weber (hoje, aliás, com crescente interesse e visibilidade nas ciências sociais em geral), têm trazido contributos que interrogam a pós-burocracia, admitindo-a parcialmente, ou, mesmo, denegando a sua centralidade, sobretudo quando estamos a assistir (paradoxalmente) à radicalização de modos de administração e gestão que devem ser considerados, mais rigorosamente, hiperburocráticos. Um interessante debate académico ainda emergente, mas que pode vir a ampliar-se, com acento, por um lado, na pós-burocracia, e, por outro, na hiperburocracia (cuja repercussão não tardará a chegar a países da lusofonia, nomeadamente ao Brasil), tem sido induzido por autores de referência nos estudos da administração educacional em Portugal. Para João Barroso, por exemplo, os "novos modos de regulação que se podem designar genericamente de pósburocráticos caracterizam-se, sobretudo, pelo facto de não se basearem, como no modelo burocrático, na legitimidade da racionalidade e da lei" (Barroso, 2013, p. 17). Por outro lado, Licínio Lima, sem deixar de sinalizar que há processos que revelam alguma erosão do modelo Weberiano ideal-típico, tem, pelo contrário, chamado a atenção para as evidências de uma "intensificação, sem precedentes, de certas dimensões burocráticas", nomeadamente pelo uso das tecnologias da informação e da comunicação de que as plataformas informáticas são um exemplo paradigmático (Lima, 2012, p. 130).

Talvez pela sua ainda recente emergência, só indiretamente se perceberão eventuais remissões para esse debate, ainda que um dos objetivos deste Dossiê temático do EPAA tenha procurado incentivar os pesquisadores e pesquisadoras de diferentes países de língua oficial portuguesa a repensar, de uma forma crítica e sustentada, empírica e/ou concetualmente, algumas das mudanças que a chamada nova gestão pública tem induzido nas práticas e conceções tradicionais da administração, direção e gestão no campo educacional, tendo em conta, sobretudo, os desafios que são colocados aos pressupostos do modelo racional-burocrático por aquela ideologia gestionária.

A expetativa era a de podermos receber contribuições que superassem alguns défices analíticos comuns na literatura, os quais traduzem, frequentemente, interpretações demasiado heterogéneas e contraditórias, ou, no mínimo, difusas, sobre a nova gestão pública. Pinçados avulsamente, alguns dos seus pressupostos, quando convocados para elucidar realidades educacionais concretas, tendem, também por isso, a reproduzir articulações insatisfatórias ou tangenciais.

Para evitar assumir a nova gestão pública como uma espécie de expressão guarda-chuva para situações muito diversas, e uma vez que muitos textos submetidos raramente retiraram consequências organizacionais, políticas e mesmo éticas da sua aplicação em contextos concretos como organizadores do dossiê optámos por dar prioridade apenas a propostas com alguma densidade relativamente a alguns dos parâmetros que tinham sido inicialmente sugeridos, ainda que com receções e tratamento muito diversos. Sobre este aspeto, por exemplo, constatamos escassa adesão em relação à sugestão que propunha que fossem consideradas as visões (ou versões) pósburocráticas e as hiperburocráticas, em articulação ou não com a nova gestão pública, muito embora, como já anteriormente aludimos, os princípios desta ideologia gestionária se confundam em algumas dimensões sobretudo com a pós-burocracia. 
No conjunto dos textos escolhidos, o que não deixa de sobressair, no entanto, são os contornos da recontextualização local, no campo educacional, da agenda global da nova gestão pública. Como chama a atenção Antoni Verger, existem múltiplas trajetórias possíveis que os modelos globais de educação sugerem, sendo por isso necessário que os pesquisadores tenham "um conhecimento aprofundado dos contextos específicos em que as políticas globais estão sendo disseminadas e adotadas", reforçando os atores, as instituições e os movimentos sociais com poderes de âgencia (agency) e de transformação (Verger, in press).

Os textos aqui reunidos tentam, em certa medida operar esse esforço, analisando políticas especificas e identificando como nesses contextos locais elas dialogam com os pressupostos veiculados por esta agenda global.

No que tange à representação da lusofonia, estão presentes neste dossiê colaborações de autores de Portugal, Brasil e Angola. Os textos abrangem o estudo de políticas voltadas para todos os níveis de ensino, da educação básica ao ensino superior, e trabalhando com fontes documentais diversas e também com diferentes atores: professores, gestores e alunos. As pesquisas analisam políticas e/ou programas específicos em cada país e em diferentes estados/regiões destes países, trazendo um mapa bastante elucidativo da diversificação e do alcance que os preceitos da nova gestão pública tem em diferentes contextos.

Scaff, Souza e Paxe fazem isso ao realizar um estudo comparado entre Brasil e Angola, a partir da análise de diferentes documentos e percebem como as reformas incidem diretamente nos modelos de gestão da educação nos dois países. Magalhães, Veiga e Videira analisam a reconfiguração do poder no ensino superior em Portugal, identificando a centralidade emergente dos gestores profissionais, ao mesmo tempo em que ocorre a diminuição do poder dos docentes na governação das respetivas instituições. Os autores apontam que essa reconfiguração se torna visível, entre outras forças, pela influência de stakeholders externos às instituições. Também contribuindo para a análise das mudanças induzidas pela nova gestão pública, Santos, Guimarães e Chaves, apresentam um estudo centrado no fenômeno da governação cooperativa nas instituições de ensino superior privadas no Brasil. Todos os estudos auxiliam a compreender esse processo de alteração nas formas conceituais, metodológicas e estruturais de se pensar a gestão.

Uma segunda tensão que os estudos abordam, diz respeito às questões relativas à avaliação e accountability. Temos aqui três diferentes estudos que auxiliam no aprofundamento das tensões e deslocamentos provocados pela nova gestão pública. Santos e Hypólito identificam, na análise de duas cidades brasileiras, que a utilização dos resultados do IDEB e da prova Brasil reforçam a implantação de mecanismos gerencialistas nas escolas. De outro lado, Oliveira e Pena, ao analisarem as percepções de professores que participam numa escola de gestores, num estado brasileiro, identificam que as avaliações externas e o Ideb causam um efeito de indução na escola, promovendo a vinculação dos professores em relação ao projeto pedagógico, aumento do número de reuniões pedagógicas e uma preocupação com o planejamento curricular. Por outro lado, as entrevistas evidenciam que o Ideb se tornou um instrumento regulatório das políticas avaliativas, voltado para a mensuração de resultados, o que contribui para a geração de um mal-estar entre os profissionais e as escolas, revelando práticas sutis e naturalizadas de uma concepção conservadora e redutora de accountability. Em seu estudo, Torres, Palhares e Afonso, identificam outros aspectos desta problemática no contexto português, afirmando que os testes estandardizados e a excelência acadêmica podem também ser relacionados e entendidos sociologicamente a partir de outros modelos e formas parcelares de accountability.

A terceira e última tensão aprofundada neste dossiê diz respeito às práticas de privatização e mercadorização que se relacionam com a nova idelogia gerencialista. Neste sentido, Cassio, Goular e Ximenes, analisam uma nova modalidade de parceria público-privada estabelecida como política no estado de São Paulo, e identificam que os chamados Contratos de Impacto Social rompem inclusive 
com os princípios de gestão democrática escolar estabelecidos na Constituição Federal. Na mesma direção, considerando o contexto do estado de Santa Catarina, Candido indica que a governança educacional, seguindo os preceitos da nova gestão pública, pretende dar um caráter mais racional às organizações educativas através das parcerias público-privadas. Por outro lado, Azevedo analisa uma experiência de "ensino público e gratuito com micro gestão privada" que aconteceu no Paraná, sustentando que o novo gerencialismo público tem similaridades com o modelo de privatização havida em Maringá com as charter schools nos EUA.

Em síntese, os textos são muito ricos em informações, experiências e reflexões a partir das recontextualizações e especificidades dos três países lusófonos referenciados. As distintas realidades podem, também por isso e no seu conjunto, exemplificar bem a direção ou os sentidos da variedade de trajetórias concretas e muiti-escalares (locais, nacionais e regionais) que existem apesar da dominância de uma agenda global para a educação. A experimentação em diferentes cenários, sobretudo de práticas contra-hegemónicas, segue sendo necessariamente um rico e urgente objeto de estudo.

\section{Referências}

Barroso, J. (2013). A emergência do local e os novos modos de regulação das políticas educativas. Educação: Temas e Problemas, 12/13, 13-25.

Barroso, J., \& Carvalho, L. M. (2011). Apontamentos sobre os «novos modos de regulação» à luz de estudos sobre as relações entre conhecimento e política. Propuesta Educativa, 36, 9-24.

Lima, L. C. (2012). Elementos de hiperburocratização da administração educacional. In: C. Lucena \& J. R. Silva Júnior (Orgs.). Trabalho e Educação no Século XXI: Experiências internacionais (pp. 129-158). São Paulo: Xamã.

Verger, A. (In press). Teorização da política de educação global: Conceitos-chave e enquadramentos. In: G. M. L. Mendes \& M. K. Borges (Orgs.). Empréstimo de Políticas Educacionais: O modelo "Um Computador Por Aluno" em Diferentes Contextos. Araraquara, SP: Junqueira \& Marin.

Verger, A., \& Curran, M. (2014). New public management as a global education policy: Its adoption and re-contextualization in a Southern European setting. Critical Studies in Education, 55(3), 253-271. http://dx.doi.org/10.1080/17508487.2014.913531 


\title{
Sobre os Autores e Editores
}

\author{
Almerindo Janela Afonso \\ Universidade do Minho - Uminho \\ ajafonso@ie.uminho.pt \\ https://orcid.org/0000-0001-9879-5814
}

Sociólogo, Doutor em Educação, Professor Associado (com tenure) da Unversidade do Minho/Portugal e pesquisador do Centro de Investigação em Educação (CIEd). É coordenador da especialidade de Sociologia da Educação e Política Educativa do doutoramento em Ciências da Educação, foi diretor do Departamento de Ciências Sociais da Educação, membro do Conselho Nacional de Educação e Presidente da Sociedade Portuguesa de Ciências da Educação.

\section{Geovana Mendonça Lunardi Mendes}

Universidade do Estado de Santa Catarina

geolunardi@gmail.com

https://orcid.org/0000-0002-8848-7436

Professora Titular do Programa de Pós-graduação em Educação, do Centro de Ciências Humanas e da Educação da Universidade do Estado de Santa Catarina(UDESC). É vice-presidente da Associação Nacional de Pesquisa e Pós-graduação em Educação(ANPED). É editora associada da Revista Brasileira de Educação, da Revista Brasileira de Educação Especial e da Arquivos Analíticos de Políticas Educativas.

\section{Dossiê Especial \\ Políticas de Administração e Gestão em Países da Lusofonia

$$
\text { arquivos analíticos de políticas educativas }
$$
Volume 26 Número 127
15 de octubro 2018
ISSN 1068-2341

\begin{abstract}
(C)
SORERIGHIS RESERVED O Copyright e retido pelo/a o autor/a (ou primeiro co-autor) que outorga o direito da primeira publicação à revista Arquivos Analíticos de Políticas Educativas. Más informação da licença de Creative Commons encontram-se em http://creativecommons.org/licenses/by-nc-nd/2.5. Qualquer outro uso deve ser aprovado em conjunto pelo/s autor/es e por AAPE/EPAA. AAPE/EPAA é publicada por Mary Lou Fulton Institute Teachers College da Arizona State University. Os textos publicados em AAPE são indexados por CIRC (Clasificación Integrada de Revistas Científicas, Espanha) DIALNET (Espanha),Directory of Open Access Journals, Education Full Text (H.W. Wilson), EBSCO Education Research Complete, ERIC, QUALIS A1 (Brasil), SCImago Journal Rank; SCOPUS, SOCOLAR (China).
\end{abstract}

Curta a nossa comunidade EPAA's Facebook https://www.facebook.com/EPAAAAPE e Twitter feed@epaa_aape. 
arquivos analíticos de políticas educativas

conselho editorial

Editor Consultor: Gustavo E. Fischman (Arizona State University)

Editoras Associadas: Kaizo Iwakami Beltrao, (Brazilian School of Public and Private Management - EBAPE/FGV, Brazil), Geovana Mendonça Lunardi Mendes (Universidade do Estado de Santa Catarina), Gilberto José Miranda, (Universidade Federal de Uberlândia, Brazil), Marcia Pletsch, Sandra Regina Sales (Universidade Federal Rural do Rio de Janeiro)

\begin{tabular}{|c|c|c|}
\hline Almerindo Afonso & Alexandre Fernandez Vaz & José Augusto Pacheco \\
\hline Universidade do Minho & Universidade Federal de Santa & Universidade do Minho, Portugal \\
\hline Portugal & Catarina, Brasil & \\
\hline Rosanna Maria Barros Sá & Regina Célia Linhares Hostins & Jane Paiva \\
\hline $\begin{array}{l}\text { Universidade do Algarve } \\
\text { Portugal }\end{array}$ & $\begin{array}{l}\text { Universidade do Vale do Itajaí, } \\
\text { Brasil }\end{array}$ & $\begin{array}{l}\text { Universidade do Estado do Rio de } \\
\text { Janeiro, Brasil }\end{array}$ \\
\hline Maria Helena Bonilla & Alfredo Macedo Gomes & Paulo Alberto Santos Vieira \\
\hline Universidade Federal da Bahia & Universidade Federal de Pernambuco & Universidade do Estado de Mato \\
\hline Brasil & Brasil & Grosso, Brasil \\
\hline Rosa Maria Bueno Fischer & Jefferson Mainardes & Fabiany de Cássia Tavares Silva \\
\hline Universidade Federal do Rio Grande & Universidade Estadual de Ponta & Universidade Federal do Mato \\
\hline do Sul, Brasil & Grossa, Brasil & Grosso do Sul, Brasil \\
\hline Alice Casimiro Lopes & Jader Janer Moreira Lopes & António Teodoro \\
\hline Universidade do Estado do Rio de & Universidade Federal Fluminense e & Universidade Lusófona \\
\hline Janeiro, Brasil & $\begin{array}{l}\text { Universidade Federal de Juiz de Fora, } \\
\text { Brasil }\end{array}$ & Portugal \\
\hline Suzana Feldens Schwertner & Debora Nunes & Lílian do Valle \\
\hline $\begin{array}{l}\text { Centro Universitário Univates } \\
\text { Brasil }\end{array}$ & $\begin{array}{l}\text { Universidade Federal do Rio Grande } \\
\text { do Norte, Brasil }\end{array}$ & $\begin{array}{l}\text { Universidade do Estado do Rio de } \\
\text { Janeiro, Brasil }\end{array}$ \\
\hline \multirow{6}{*}{$\begin{array}{l}\text { Flávia Miller Naethe Motta } \\
\text { Universidade Federal Rural do Rio de } \\
\text { Janeiro, Brasil }\end{array}$} & Alda Junqueira Marin & \multirow{6}{*}{$\begin{array}{l}\text { Alfredo Veiga-Neto } \\
\text { Universidade Federal do Rio Grande } \\
\text { do Sul, Brasil }\end{array}$} \\
\hline & Pontifícia Universidade Católica de & \\
\hline & São Paulo, Brasil & \\
\hline & Dalila Andrade Oliveira & \\
\hline & Universidade Federal de Minas & \\
\hline & Gerais, Brasil & \\
\hline
\end{tabular}


archivos analíticos de políticas educativas
consejo editorial

Editor Consultor: Gustavo E. Fischman (Arizona State University)

Editores Asociados: Armando Alcántara Santuario (Universidad Nacional Autónoma de México), Ezequiel Gomez

Caride (Pontificia Universidad Católica Argentina), Antonio Luzon (Universidad de Granada), Angelica Buendia

(Metropolitan Autonomous University), José Luis Ramírez (Universidad de Sonora), Paula Razquin (Universidad de San Andrés)

\section{Claudio Almonacid}

Universidad Metropolitana de

Ciencias de la Educación, Chile

\section{Miguel Ángel Arias Ortega \\ Universidad Autónoma de la \\ Ciudad de México \\ Xavier Besalú Costa \\ Universitat de Girona, España \\ Xavier Bonal Sarro Universidad \\ Autónoma de Barcelona, España}

Antonio Bolívar Boitia

Universidad de Granada, España

José Joaquín Brunner Universidad

Diego Portales, Chile

Damián Canales Sánchez

Instituto Nacional para la

Evaluación de la Educación,

México

Gabriela de la Cruz Flores

Universidad Nacional Autónoma de México

Marco Antonio Delgado Fuentes

Universidad Iberoamericana,

México

Inés Dussel, DIE-CINVESTAV, México

Pedro Flores Crespo Universidad

Iberoamericana, México

\author{
Ana María García de Fanelli \\ Centro de Estudios de Estado y \\ Sociedad (CEDES) CONICET, \\ Argentina \\ Juan Carlos González Faraco \\ Universidad de Huelva, España \\ María Clemente Linuesa \\ Universidad de Salamanca, España \\ Jaume Martínez Bonafé \\ Universitat de València, España
}

Alejandro Márquez Jiménez

Instituto de Investigaciones sobre la

Universidad y la Educación,

UNAM, México

María Guadalupe Olivier Tellez,

Universidad Pedagógica Nacional,

México

Miguel Pereyra Universidad de

Granada, España

Mónica Pini Universidad Nacional

de San Martín, Argentina

Omar Orlando Pulido Chaves

Instituto para la Investigación

Educativa y el Desarrollo

Pedagógico (IDEP)

José Ignacio Rivas Flores

Universidad de Málaga, España

\author{
Miriam Rodríguez Vargas \\ Universidad Autónoma de \\ Tamaulipas, México \\ José Gregorio Rodríguez \\ Universidad Nacional de Colombia, \\ Colombia \\ Mario Rueda Beltrán Instituto de \\ Investigaciones sobre la Universidad \\ y la Educación, UNAM, México \\ José Luis San Fabián Maroto \\ Universidad de Oviedo, \\ España
}

Jurjo Torres Santomé, Universidad de la Coruña, España

Yengny Marisol Silva Laya

Universidad Iberoamericana,

México

Ernesto Treviño Ronzón

Universidad Veracruzana, México

\section{Ernesto Treviño Villarreal \\ Universidad Diego Portales \\ Santiago, Chile}

Antoni Verger Planells

Universidad Autónoma de

Barcelona, España

Catalina Wainerman

Universidad de San Andrés, Argentina

Juan Carlos Yáñez Velazco

Universidad de Colima, México 
education policy analysis archives editorial board

Lead Editor: Audrey Amrein-Beardsley (Arizona State University)

Editor Consultor: Gustavo E. Fischman (Arizona State University)

Associate Editors: David Carlson, Lauren Harris, Eugene Judson, Mirka Koro-Ljungberg, Molly Ott, Scott Marley, Iveta Silova (Arizona State University)

Cristina Alfaro San Diego State

University

Gary Anderson New York University

Michael W. Apple University of Wisconsin, Madison

Jeff Bale OISE, University of Toronto, Canada

Aaron Bevanot SUNY Albany

David C. Berliner Arizona

State University

Henry Braun Boston College

Casey Cobb University of

Connecticut

Arnold Danzig San Jose State

University

Linda Darling-Hammond

Stanford University

Elizabeth H. DeBray University of Georgia

Chad d'Entremont Rennie Center for Education Research \& Policy

John Diamond University of Wisconsin, Madison

Matthew Di Carlo Albert Shanker Institute

Sherman Dorn Arizona State University

Michael J. Dumas University of California, Berkeley

Kathy Escamilla University of Colorado, Boulder

Melissa Lynn Freeman Adams State College

Rachael Gabriel

University of Connecticut

Amy Garrett Dikkers University of North Carolina, Wilmington
Gene V Glass Arizona

State University

Ronald Glass University of

California, Santa Cruz

Jacob P. K. Gross University of Louisville

Eric M. Haas WestEd

Julian Vasquez Heilig California State University, Sacramento

Kimberly Kappler Hewitt University of North Carolina Greensboro Aimee Howley Ohio University

Steve Klees University of Maryland

Jaekyung Lee

SUNY Buffalo

Jessica Nina Lester

Indiana University

Amanda E. Lewis University of Illinois, Chicago

Chad R. Lochmiller Indiana

University

Christopher Lubienski Indiana University

Sarah Lubienski Indiana University

William J. Mathis University of Colorado, Boulder

Michele S. Moses University of

Colorado, Boulder

Julianne Moss Deakin

University, Australia

Sharon Nichols University of Texas, San Antonio

Eric Parsons University of Missouri-Columbia

Amanda U. Potterton University of Kentucky
Susan L. Robertson Bristol

University, UK

Gloria M. Rodriguez

University of California, Davis

R. Anthony Rolle University of Houston

A. G. Rud Washington State University

Patricia Sánchez University of University of Texas, San Antonio

Janelle Scott University of California, Berkeley Jack Schneider University of Massachusetts, Lowell

Noah Sobe Loyola University

Nelly P. Stromquist University of Maryland

Benjamin Superfine University of Illinois, Chicago

Adai Tefera Virginia

Commonwealth University

Tina Trujillo University of

California, Berkeley

Federico R. Waitoller University of Illinois, Chicago

Larisa Warhol

University of Connecticut

John Weathers University of Colorado, Colorado Springs

Kevin Welner University of Colorado, Boulder

Terrence G. Wiley Center for Applied Linguistics

John Willinsky

Stanford University

Jennifer R. Wolgemuth University of South Florida

Kyo Yamashiro Claremont Graduate University 